\title{
Tilt of sunspot bipoles in Solar Cycles 15 to 24
}

\author{
Ksenia Tlatova ${ }^{1}$ (ID) Andrey Tlatov $^{2,3}$ (D) \\ Alexei Pevtsov ${ }^{4,5}$. Kalevi Mursula ${ }^{5}$ (D) \\ Valeria Vasil'eva ${ }^{2}$. Elina Heikkinen ${ }^{5}$. \\ Luca Bertello ${ }^{4}$. Alexander Pevtsov ${ }^{4,5}$. \\ Ilpo Virtanen ${ }^{5}$ (D) Nina Karachik ${ }^{6}$
}

Received: 24 February 2018 / Accepted: 20 July 2018 / Published online:

\author{
$凶$ A.A. Pevtsov \\ apevtsov@nso.edu \\ K. A. Tlatova \\ k.tlatova@mail.ru \\ A.G. Tlatov \\ tlatov@mail.ru \\ K. Mursula \\ kalevi.mursula@oulu.fi \\ E. Heikkinen \\ elina.heikkinen@oulu.fi \\ L. Bertello \\ Ibertello@nso.edu \\ A.A. Pevtsov \\ apevtsov@nmsu.edu \\ I.I. Virtanen \\ ilpo.virtanen@oulu.fi \\ N.V. Karachik \\ ninakarachik@mail.ru \\ 1 St. Petersburg State University, Saint Petersburg, Russian Federation \\ 2 Kislovodsk Mountain Astronomical Station of Pulkovo Observatory, Kislovodsk, \\ Russian Federation \\ 3 Kalmyk State University, Elista, 358009, Russian Federation \\ 4 National Solar Observatory, 3665 Discovery Drive, 3rd Floor, Boulder, CO 80303 \\ USA \\ 5 ReSoLVE Centre of Excellence, Space Climate research unit, University of Oulu, \\ POB 3000, FIN-90014, Oulu, Finland \\ 6 Astronomical Institute AS RUz, 33 Astronomicheskaya ul., Tashkent, 100052, \\ Uzbekistan
}




\begin{abstract}
We use recently digitized sunspot drawings from Mount Wilson Observatory to investigate the latitudinal dependence of tilt angles of active regions and its change with solar cycle. The drawings cover the period from 1917 to present and contain information about polarity and strength of magnetic field in sunspots. We identify clusters of sunspots of same polarity, and used these clusters to form "bipole pairs". The orientation of these bipole pairs was used to measure their tilts. We find that the latitudinal profile of tilts does not monotonically increase with latitude as most previous studies assumed, but instead, it shows a clear maximum at about 25-30 degree latitudes. Functional dependence of tilt $(\gamma)$ on latitude $(\varphi)$ was found to be $\gamma=(0.20 \pm 0.08) \sin (2.80 \varphi)+(-0.00 \pm 0.06)$. We also find that latitudinal dependence of tilts varies from one solar cycle to another, but larger tilts do not seem to result in stronger solar cycles. Finally, we find the presence of a systematic offset in tilt of active regions (non-zero tilts at the equator), with odd cycles exhibiting negative offset and even cycles showing the positive offset.
\end{abstract}

Keywords: Sun: activity - Sun: magnetic fields - sunspots

\title{
1. Introduction
}

Orientation of solar bipoles, as defined by a line connecting their leading and following parts, exhibits a slight systematic tilt relative to the direction of solar circles of constant latitude (parallels) with trailing part being situated at higher latitudes as compared with their leading part. Tilt angles show a tendency to increase with the latitude. This pattern, dubbed "Joy's law" by H. Zirin (1988), was first described by Hale et al. (1919). In the framework of surface flux transport models, the active region tilt is one of the important ingredients that affects the strength of polar field and therefore, is important for the amplitude of the next solar magnetic cycle (e.g., Cameron et al., 2010). Joy's law was extensively studied using both white light and magnetic field observations (e.g., Howard, 1991; Sivaraman, Gupta, and Howard, 1999; McClintock, Norton, and Li, 2014; McClintock and Norton, 2016, for review, see Pevtsov et al., 2014). Current interpretations of active regions' tilt include three underlining causes: toroidal field orientation (e.g., Babcock, 1961; Norton and Gilman, 2005), action of the Coriolis force (e.g., Fisher et al., 2000), and signature of kink-instability (e.g., Leighton, 1969; Longcope et al., 1999; Holder et al., 2004).

Unlike the model predictions, the observational evidence about the importance of the tilt angles for determining the strength of the next solar cycle is inconclusive. Several authors (e.g., Sivaraman, Gupta, and Howard, 1999; Dasi-Espuig et al., 2010) reported that for Cycles 16-21 the average tilt angles, normalized by latitude, correlate with the amplitude of the next solar cycle. On the other hand, later studies (e.g., Ivanov, 2012; McClintock and Norton, 2013) could not reproduce the results of Dasi-Espuig et al. (2010), and later, DasiEspuig et al. (2013) revised their earlier results. McClintock and Norton (2013) indicated that cycle variation of tilt angles may differ for the two solar hemispheres. Indeed, Li and Ulrich (2012) found the least-square linear fit to their 
measurements of tilts derived from magnetogram data during 1974-2013 (Cycles $21-24)$ as $\gamma=(0.5 \pm 0.2) \varphi-\left(0.9^{\circ} \pm 0.3^{\circ}\right)$. They concluded that the observed non-zero offset reflects the asymmetry of tilts between Southern and Northern hemispheres. The dependency of tilt $(\gamma)$ on latitude $(\varphi)$ is typically fitted by a function of latitude, $\gamma \propto f(\varphi)$ or sine of latitude, $\gamma \propto f(\sin \varphi)$. Functional dependency is often (but not always, see examples in Table 1 in Pevtsov et al., 2014) assumed to have a zero tilt angle at the equator. $\gamma \propto f(0) \equiv 0$. Pevtsov et al. (2014, and references therein) noted that both of these assumptions may require additional studies: some datasets appear showing a maximum tilt angle in some latitudes, and when the fit is not constrained by the assumption of zero crossing at the equator, it often shows a non-zero offset. The presence of such an offset may indicate that our present understanding of tilt of active regions is incomplete.

In this paper, we use the magnetic field observations in sunspots from the Mount Wilson Observatory (MWO) to further investigate the latitudinal dependence of tilt angles in Cycles 15-24. In Section 2 we describe our dataset and the approach in determining the proxy for tilt angles. Section 3 discusses variation of tilt with solar latitude for both hemispheres. In Section 4 we compare latitudinal variation of tilt for different solar cycles, and in Section 5 we discuss our findings.

\section{Data}

We use data derived from daily sunspot drawings taken at Mount Wilson Observatory (MWO). This MWO program started in early 1917, and it continues till present with some short interruptions in more recent years due to funding shortages. A typical drawing (for a graphic example, see Figure 1 in Pevtsov and Clette, 2014) contains information about location of sunspot, its approximate size (penumbra and umbra) as well as polarity and maximum field strength of its magnetic field. The drawings were digitized (tabulated) using software package developed by us (e.g., Tlatova, Vasil'eva, and Pevtsov, 2015). The digitization included the date and time of observations, heliographic coordinates of each umbra, its area, the strength, and polarity of its magnetic field. The digitized dataset employed in this article contains 20318 days of observations, which cover period between January 1917 and October 2016. Total number of features (sunspots and pores) on these images is about $5 \times 10^{5}$.

The results of the initial digitization (version 2017_11_01_AT_mwo, used here) may contain small number of errors related to identification of solar limbs on drawings, incorrect time stamps, and in rare cases, incorrect polarity recordings from the original drawings. Such errors are not unusual given the size of our dataset and various subjective factors related to both observations (multiple observers with different hand-writing and using different recording techniques) and manual digitization. A detailed study to quantify and correct some of these errors is currently underway (Pevtsov et al. 2018, in preparation), but it is clear that the effect of these errors is rather small.

One clear advantage of the MWO sunspot drawings as compared with all previous studies of tilt angles using historical data is that knowing the polarities 
of sunspots allows a better determination of sunspot pairs forming a group. Pevtsov et al. (2014) provided examples of group mis-identification when only white light images are used.

Sunspot groups are identified on the original MWO drawings by the MWO group number and heliographic coordinates of the center of the group, but these data were not included in the initial digitization used here. Hence, we turned to an alternative procedure of identifying bipole pairs of opposite polarity. The detailed discussion of early version of this algorithm is presented in Tlatov, Vasil'eva, and Pevtsov (2010). We slightly modified the algorithm by adding a step to identify clusters of sunspots of positive and negative polarity. To identify a cluster, we start from the largest (by area) sunspot of corresponding (say, positive) polarity, and search for same polarity sunspots within 10 degrees in longitude and 7 degrees in latitude. The heliographic coordinates of center of each cluster are computed as mean location of all sunspots within cluster weighted by their areas. The clusters are used to form bipolar pairs. At this step, the algorithm starts from the center of an arbitrary cluster of positive polarity and searches for a negative polarity cluster, whose center is at the distance within 15 degrees in longitude and 7 degrees in latitude. The procedure is repeated to search for a closest cluster of positive polarity using as starting point cluster of negative polarity identified on previous step. If the closest positive polarity cluster found on this step coincides with the starting positive polarity cluster, both positive and negative polarity clusters are marked as a pair, and removed from the list of elements for future searches. Similar procedures had been successfully used in several past studies (e.g., Sattarov et al., 2002). Once the bipole pair is identified, the tilt angle of its magnetic axis with respect to the constant latitude circle is calculated. We adopted a "classical" definition of active region tilt as angle between the line connecting leading and trailing polarity sunspots of a group and the solar equator. This definition corresponds to Figure $1 \mathrm{~b}$ in $\mathrm{Li}$ and Ulrich (2012). In this reference frame, active regions following the Joy's law will have positive/negative tilt in the northern/southern hemisphere. Mean latitude (and longitude) of groups was determined as the mean of heliographic coordinates of two clusters comprising the group.

Figure 1 provides examples of clusters of sunspots and corresponding tilts determined using our approach. In this Figure the reader may see examples of active regions, whose tilt follows Joy's law (e.g., group 11499) or deviates from it (group 11498). There are also cases, when sunspots were not selected by our routine for tilt determination. For example, non-numbered (NN) pore at S27W33 does not have polarity information. Group 11496 at N32E42 has its leading polarity pore marked as "R faint", but this pore was not included in the digitized set leaving the negative $(\mathrm{V})$ polarity pores without a bipolar pair.

Each drawing was treated independently of all others. Thus, for example, if an active region persisted for several days, the dataset will include multiple tilt measurements for this region. This approach may bias long-lived groups. Furthermore, the tilt of an active region may change with time depending on total magnetic flux/area of bipoles (McClintock and Norton, 2016). Not all features identified on the drawings will contribute to "bipole pairs" (for comparison, our data set contains about $5 \times 10^{5}$ individual flux elements, but only about $5 \times 10^{4}$ 


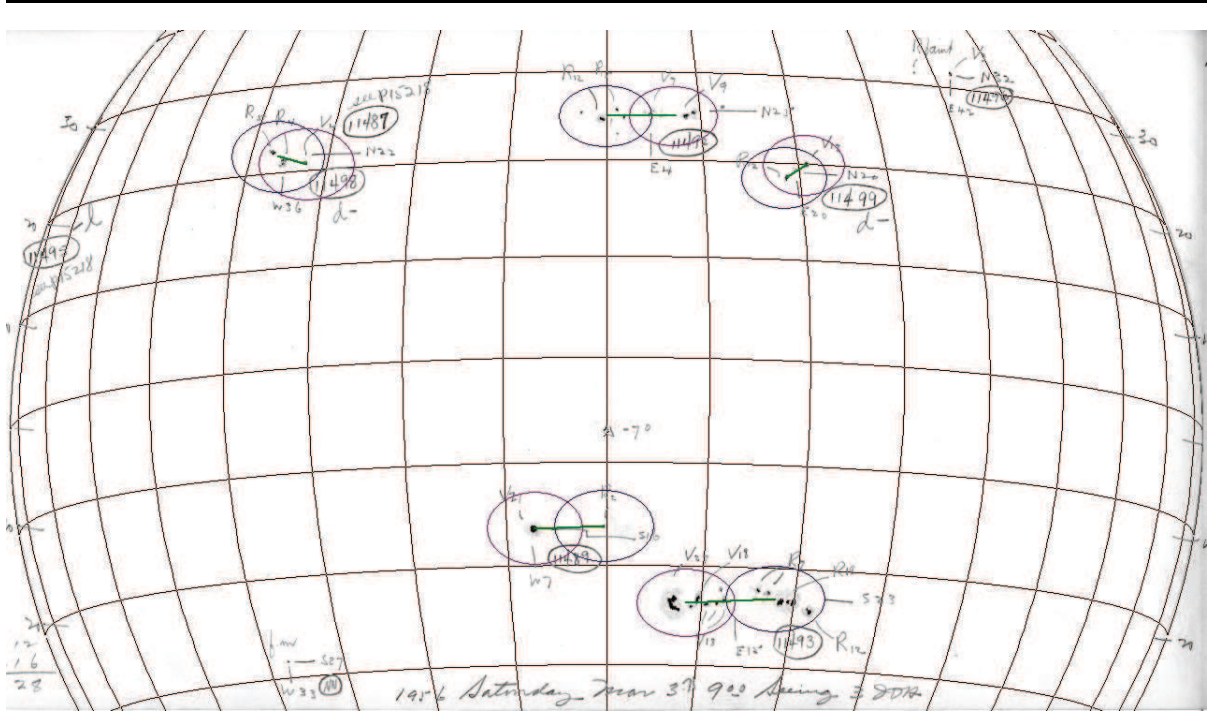

Figure 1. Example of bipole pairs identified by our algorithm for observations taken on 3 March 1956. Letters V/R corresponds to negative/positive polarities, and the numeric value after the letter corresponds to measured field strength in units of hundred Gauss. For example, V27 corresponds to negative field strength of 2700 Gauss. Clusters of positive/negative sunspots are outlined by purple/blue ovals. Green line segments correspond to magnetic axis of each group as determined by our routine. Image is oriented with North up and East to the right.

bipoles). Nevertheless, we expect that the average orientation of individual pairs belonging to the same group would be close to an average orientation of the group. Using sunspot drawings for tilt studies has some limitations. For example, groups with highly asymmetric polarity distributions (unipolar sunspots) will be excluded. Also, drawings are not inclusive. Some features (small pores) may be present on a drawing, but have no corresponding magnetic field measurements. Such features will not be included in our determination of tilt.

\section{Latitudinal Profile of Active Region Tilts}

Next, we divided all identified bipoles in 10-degree latitudinal intervals, and used the distributions of tilts in each interval to derive the mean tilt and its standard deviation. This approach takes into account any change in tilt angle with age of an active region. The latitudinal intervals were spaced by 5 degree in latitudes and thus, neighboring intervals overlap by 5 degrees (e.g., $40^{\circ} \pm 5^{\circ}$, $35^{\circ} \pm 5^{\circ}, 30^{\circ} \pm 5^{\circ}$ etc). Figure 2 provides example of distribution of tilts for three latitudinal ranges: $5^{\circ} \pm 5^{\circ}, 15^{\circ} \pm 5^{\circ}$ and $25^{\circ} \pm 5^{\circ}$, as well as the corresponding fits of these distributions by the Gaussian functions. Mean tilts and their standard deviations derived that way are listed in Table 1. Figure 3 shows the tilts for all years (see columns 2 and 3 of Table 1) in graphical form.

Similar to previous studies (for review, see Pevtsov et al., 2014) the tilt angles of bipoles in our dataset exhibit a slight dependence on latitude in their tilt angles 


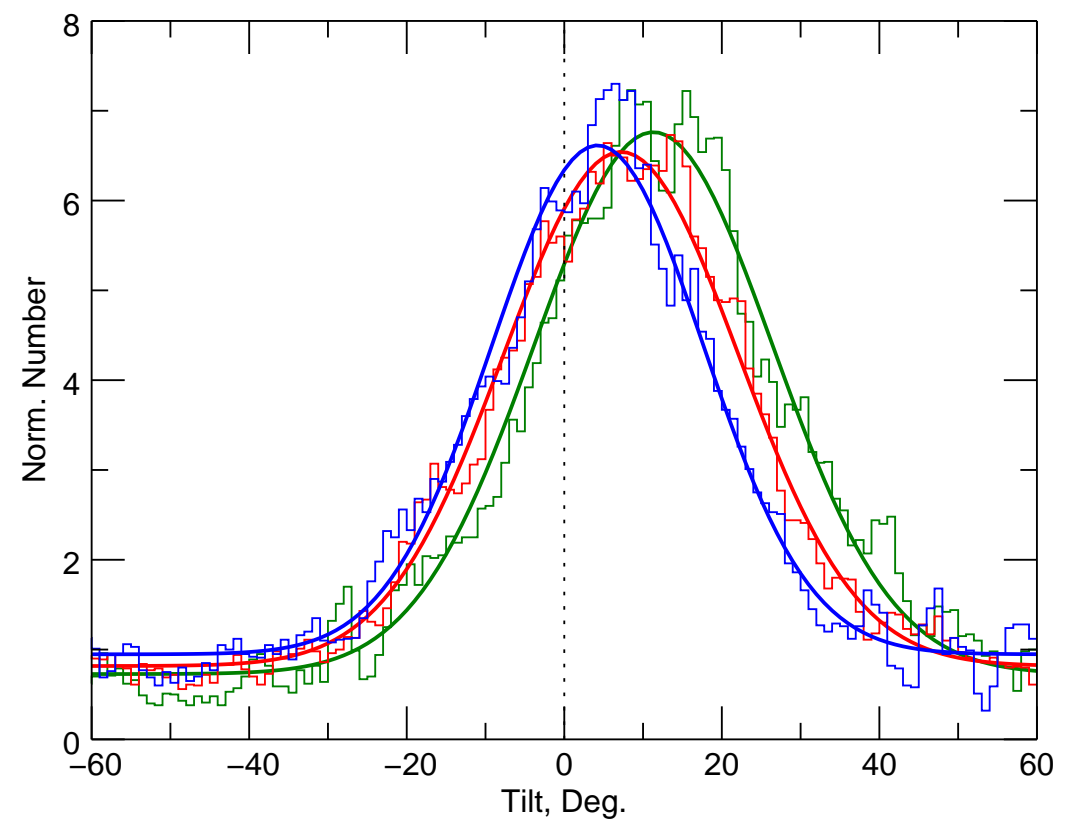

Figure 2. Distribution of tilt angles for three latitudinal ranges in the Northern hemisphere: $0-10^{\circ}$ (blue), $10-20^{\circ}$ (red), and $20-30^{\circ}$ (green). Thin lines show histograms of tilts at one-degree bins, and solid thick lines are Gaussian fits to the distributions. For a reference, vertical dotted line corresponds to zero mean. To simplify their visual comparison, all three distributions are scaled to be about the same maximum amplitude.

with a significant scatter. Best linear fit to our data (using standard deviations as statistical uncertainties, returns

$$
\gamma=(0.41 \pm 0.18) \varphi+(0.00 \pm 0.06)
$$

where the coefficients are expressed in radians for better comparison with the summary Table 1 in Pevtsov et al. (2014). This fit is shown in Figure 3 by dashed line. The coefficients (slopes) are similar (albeit slightly larger) to Fisher, Fan, and Howard (1995); Dasi-Espuig et al. (2010); Ivanov (2012), and the fitted slope is significantly smaller in comparison with Stenflo and Kosovichev (2012), (for review, see summary Table 1 in Pevtsov et al., 2014). Stenflo and Kosovichev (2012) fit (shown as a dotted curve in Figure 3) appears to match our data quite well at low latitudes, but at latitudes higher that 20 degrees the fitted curve deviates significantly from the observations. Finally, we found that the data shown in Figure 3 are best represented by

$$
\gamma=(0.20 \pm 0.08) \sin (2.80 \varphi)+(-0.00 \pm 0.06),
$$


Table 1. Parameters of Gaussian distribution of bipole tilts for selected latitudinal ranges and separately for odd and even cycles

\begin{tabular}{r|rrrrrrrr}
\hline \multirow{2}{*}{$\begin{array}{r}\text { Latitude } \\
\text { degree }\end{array}$} & \multicolumn{3}{|c}{ All Cycles } & \multicolumn{3}{c}{ Odd Cycles } & \multicolumn{3}{c}{ Even Cycles } \\
& $\gamma^{a}$ & $\sigma^{b}$ & $\gamma^{a}$ & $\sigma^{b}$ & $N^{c}$ & $\gamma^{a}$ & $\sigma^{b}$ & $N^{c}$ \\
\hline 35 & 8.77 & 15.59 & 7.20 & 22.70 & 329 & 12.30 & 30.30 & 413 \\
30 & 11.19 & 14.62 & 10.60 & 26.40 & 1142 & 13.40 & 24.50 & 1251 \\
25 & 11.31 & 15.14 & 11.40 & 24.40 & 2699 & 12.10 & 24.10 & 2780 \\
20 & 8.94 & 15.72 & 9.70 & 24.80 & 4915 & 9.90 & 24.00 & 4603 \\
15 & 7.20 & 14.89 & 7.90 & 23.40 & 6374 & 7.60 & 23.20 & 5846 \\
10 & 6.16 & 14.03 & 7.10 & 21.50 & 5363 & 5.40 & 21.20 & 5128 \\
5 & 4.23 & 13.43 & 6.20 & 21.70 & 2984 & 3.10 & 21.20 & 2643 \\
0 & -0.39 & 11.80 & 5.50 & 24.00 & 1465 & -3.60 & 23.50 & 1373 \\
-5 & -2.23 & 13.85 & -0.30 & 22.60 & 2840 & -5.10 & 20.30 & 3088 \\
-10 & -4.89 & 13.73 & -4.50 & 22.50 & 5406 & -5.80 & 21.20 & 5668 \\
-15 & -6.77 & 14.28 & -6.80 & 22.30 & 6045 & -7.40 & 23.40 & 6069 \\
-20 & -8.63 & 14.91 & -8.80 & 22.30 & 4903 & -9.60 & 26.80 & 4178 \\
-25 & -10.94 & 13.86 & -11.70 & 22.20 & 3100 & -11.00 & 26.00 & 1991 \\
-30 & -12.13 & 12.93 & -13.30 & 22.10 & 1429 & -11.10 & 23.00 & 812 \\
-35 & -12.79 & 12.27 & -14.00 & 23.10 & 384 & -10.10 & 21.40 & 295 \\
\hline
\end{tabular}

(a) Average tilt angle

(b) Standard deviation

(c) Number of data points

where $\gamma$ and $\varphi$ are in radians. This fit is shown in Figure 3 by a solid curve. Here the 2.80 coefficient is also determined, together with the other two coefficients, via multidimensional minimization, using the downhill simplex method ("AMOEBA" routine, Press et al., 1992).

\section{Latitudinal variation of tilt for odd and even cycles}

Our dataset allows investigating possible variations in Joy's law with the solar cycle. The latitude, at which the solar cycle starts, correlates with its cycle amplitude (see, e.g. Tlatov and Pevtsov, 2010): high amplitude cycles have sunspots appearing first at higher latitudes as compared to cycles with lower amplitude. Normalizing tilt angles by latitude would then reduce this dependence (e.g., $\frac{\gamma}{\varphi}=\frac{A \sin \varphi}{\varphi}+\frac{B}{\varphi} \approx \frac{A \varphi}{\varphi}=A$. Thus, coefficient A (slope of tilt vs. latitude dependence) can be used to investigate the observational evidence of dependence of tilt on strength of solar cycle. Figure 4 shows latitudinal tilt profiles for individual cycles. Within the latitude range of $\pm 10-25^{\circ}$, the tilt profiles appear to be about the same for Cycles 15-24. At high latitudes, the data show significant scatter and large variations between different cycles. Coefficients for Equation 1 fitted to individual cycles confirm this visual impression (see, columns 2 and 3 Table 3, To mitigate the differences in tilts at high- and lowlatitudes, for this test, the fitting was limited to tilts at $\pm 10-25^{\circ}$ latitudinal 


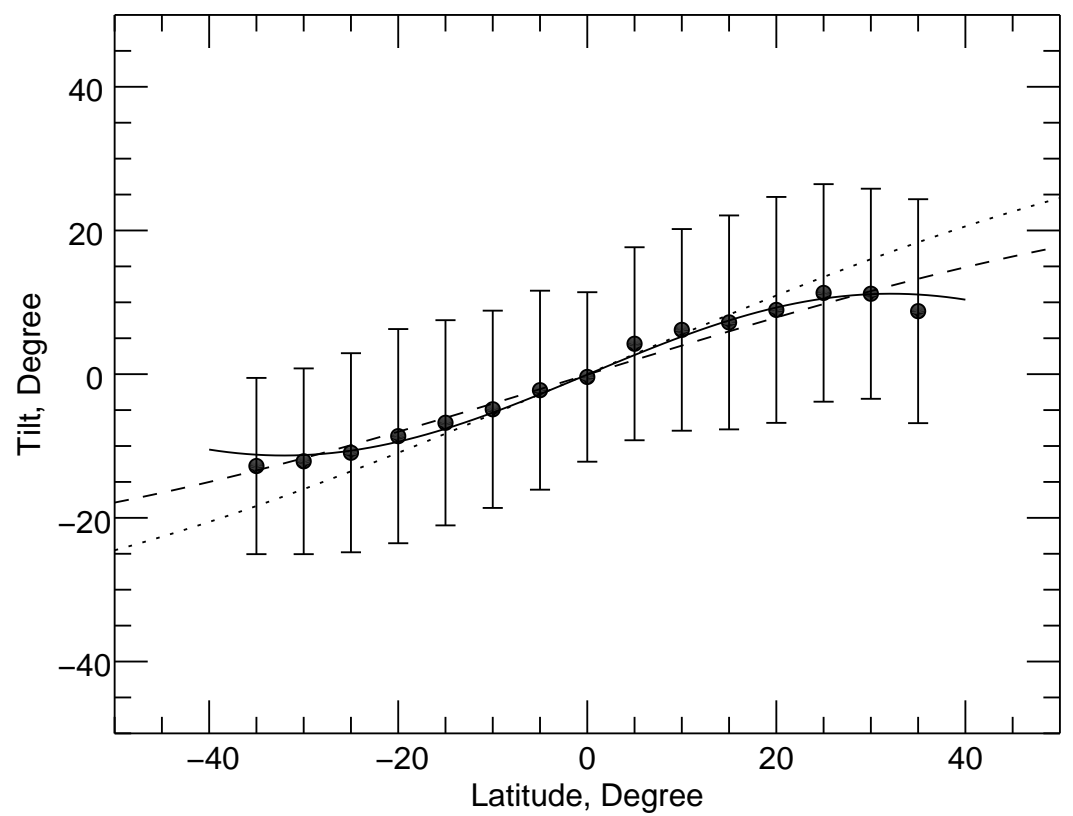

Figure 3. Mean tilts of magnetic bipoles (filled circles) and their standard deviations (error bars) for all cycles. Solid curve corresponds to sine fit by Equation 2 dashed line is fit by Equation 1 and dotted curve represents sine fit by Stenflo and Kosovichev (2012).

ranges). Indeed, the tilt dependence on latitude does differ for different cycles. For example, on average, the latitudinal dependence is slightly steeper for Cycles 15, 16, 18, 20, 21-23 (group 1) as compared to Cycles 17, 19, and 24 (group 2). However, within each group the difference in A (slope) is about the same, and the difference between two groups is not too large. Furthermore, the data show no clear pattern implying the presence of a relation between larger A of Cycle $n$ and the strength of Solar Cycle $(n+1)$. For example, Cycles 22 and 23 have the steepest latitudinal dependence of all cycles, but neither was followed by a particular strong (in amplitude) sunspot cycle. Spearman's rank correlation $\tau$ between A-coefficient in Cycle $(n)$ and sunspot number (SSN) in Cycle $(n+1)$ is even negative, $\tau=-0.409$ with significant chance of random occurrence 0.25.

One interesting result can be noticed in Figure 4 at low latitudes: odd cycles seem to exhibit a positive offset in their zero-latitude tilt, while for the even cycles the offset is negative. The pattern can also be identified in Table 2 in tilts at zero latitude. Figure 5 shows average latitudinal profile of tilt angles computed separately for even and odd cycles. The difference barely exceeds one sigma statistical significance level, but it does appear non-random. 


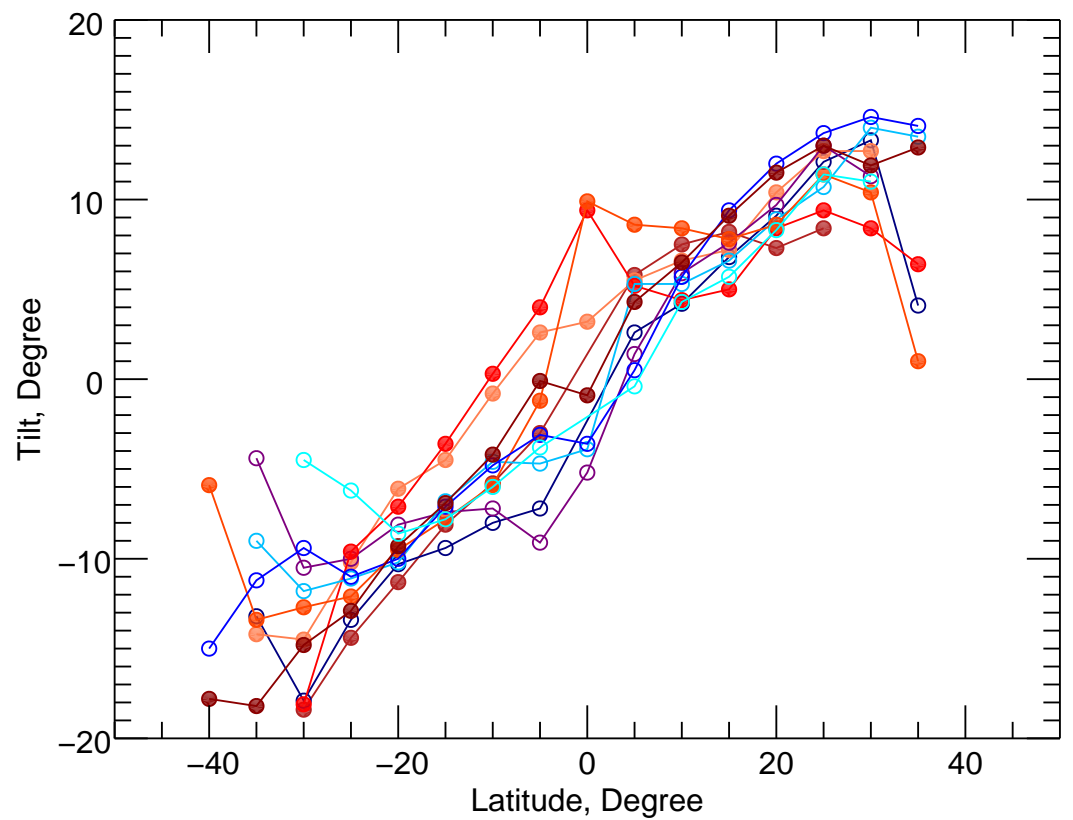

Figure 4. Latitudinal profiles of tilts for odd (filled circles, red-hued color) and even (open circles, blue-hued colors). The data come from Table 2

\section{Discussion}

The orientation (tilt) of active regions is one of critical parameters that affects the poleward transport of magnetic field originating from decaying active regions. In the framework of surface flux transport models, which are increasingly used in evaluating the amplitude of future solar cycles, active region tilt is one of the important ingredients that affects the strength of polar field. In its turn, the strength of polar field determines the amplitude of next solar cycle (e.g.,Baumann et al., 2004; Cameron et al., 2010). Thus, a proper determination of latitudinal variation of active region tilt angles, and its possible change from cycle to cycle are important for understanding and modeling future sunspot activity cycles. Our study revealed several previously unknown tendencies in the variation of the tilt angles of solar active regions with latitude (so called Joy's law): the presence of a maximum at about $25-30^{\circ}$ range of latitudes and an opposite offset in non-zero tilt at the equator for odd and even cycles.

In some previous studies, one of the unspoken assumptions was that the functional dependence of tilts is a function $\mathrm{f}(\varphi)$, which monotonically increases with latitude. Some studies used $\mathrm{f}(\varphi) \propto \varphi$ or $\mathrm{f}(\varphi) \propto \sin \varphi$. By itself, the assumption is reasonable, if one accepts that the action of the Coriolis force is the underlying explanation for this solar phenomenon. In fact, the combination 


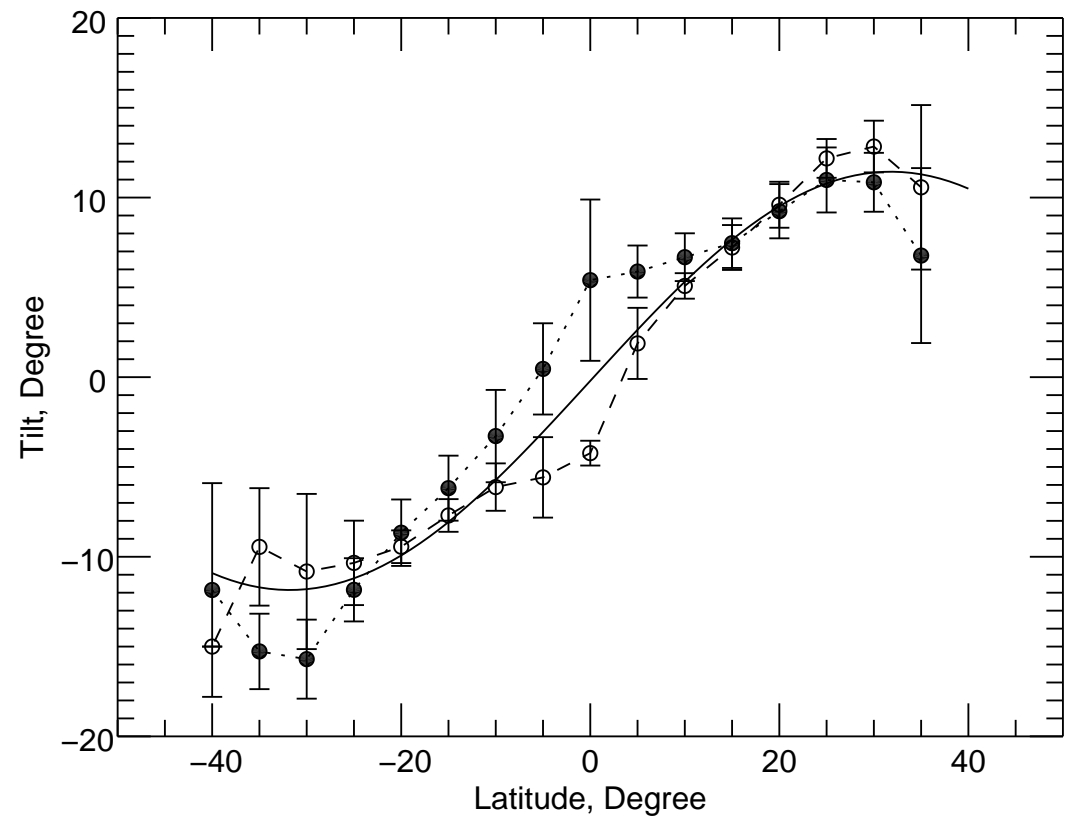

Figure 5. Mean tilts of magnetic bipoles for odd (filled circles, dotted line) and even (open circles, dashed line). Solid line represents fit to all dataset shown in Figure 2

of the Coriolis force and fluxtube dynamics (e.g., material drainage, significant scatter presumably caused by interaction with turbulent convection) seems to explain the previously observed properties of active region tilts reasonable well (Fisher et al., 2000).

However, the non-monotonic behavior in tilt angle with latitudes described in this paper, may still be explained in the framework of Fisher, Fan, and Howard (1995) model. On the basis of a model of a thin flux tube, whose main axis is distorted by the action of the Coriolis force and by the interaction with turbulent convection, Fisher, Fan, and Howard (1995) derived a dependence of tilt of active regions on latitude and footpoint separation. This dependence may, in fact, offer at least a quantitative explanation for our observation of a non-monotonic behavior of tilt with latitude. Active regions emerging at the beginning of each solar cycle, are typically smaller in size and they emerge at higher latitudes. The main activity in each cycle develops in lower latitudes with large regions usually emerging around the maximum of cycle or slightly after it. This range of latitudes will exhibit the largest tilt. As smaller regions tend to exhibit smaller tilt, those regions that emerge at higher latitudes at the beginning of the cycle will exhibit tendency for smaller tilt, as compared with the larger regions in lower latitudes that emerge later in the cycle. The number of active regions at high latitudes is significantly smaller as compared to lower latitudes 
Table 2. Mean tilts for latitudinal ranges for Cycles 15-24

\begin{tabular}{r|rrrrrrrrrr}
\hline \multirow{2}{*}{ Latitude } & C15 & C16 & C17 & C18 & C19 & C20 & C21 & C22 & C23 & C24 \\
\hline 35 & & 4.1 & & & 6.4 & 13.5 & 1.0 & 14.1 & 12.9 & \\
30 & & 13.3 & 12.7 & 11.3 & 8.4 & 14.0 & 10.40 & 14.6 & 11.9 & 11.0 \\
25 & 8.4 & 12.1 & 12.70 & 13.0 & 9.4 & 10.7 & 11.4 & 13.7 & 13.0 & 11.4 \\
20 & 7.3 & 9.1 & 10.4 & 9.7 & 8.4 & 8.9 & 8.6 & 12.0 & 11.5 & 8.3 \\
15 & 8.2 & 6.8 & 7.2 & 7.6 & 5.0 & 6.6 & 7.80 & 9.4 & 9.1 & 5.7 \\
10 & 7.5 & 4.2 & 6.6 & 5.9 & 4.4 & 5.3 & 8.4 & 5.7 & 6.5 & 4.3 \\
5 & 5.8 & 2.6 & 5.5 & 1.4 & 5.2 & 5.3 & 8.6 & 0.5 & 4.3 & -0.4 \\
0 & & & 3.2 & -5.2 & 9.4 & -3.9 & 9.9 & -3.6 & -0.9 & \\
-5 & -3.0 & -7.2 & 2.6 & -9.1 & 4.0 & -4.7 & -1.2 & -3.1 & -0.1 & -3.8 \\
-10 & -5.8 & -8.0 & -0.8 & -7.2 & 0.3 & -4.6 & -5.9 & -4.8 & -4.2 & -6.0 \\
-15 & -8.1 & -9.4 & -4.5 & -7.4 & -3.6 & -6.8 & -7.8 & -7.1 & -6.9 & -7.8 \\
-20 & -11.3 & -10.3 & -6.1 & -8.1 & -7.1 & -10.2 & -9.5 & -10.0 & -9.3 & -8.6 \\
-25 & -14.4 & -13.4 & -10.2 & -10.0 & -9.6 & -11.1 & -12.1 & -11.0 & -12.9 & -6.2 \\
-30 & -18.4 & -17.9 & -14.5 & -10.5 & -18.1 & -11.8 & -12.7 & -9.4 & -14.8 & -4.5 \\
-35 & & -13.2 & -14.2 & -4.4 & & -9.0 & -13.4 & -11.2 & -18.2 & -17.8 \\
-40 & & & & & & & -5.9 & -15.0 & - \\
\hline
\end{tabular}

(see, Table 1. columns 6 and 9), which increases the statistical uncertainty of tilt angles for high latitudes. We think that a combination of these two aspects could explain the appearance of a maximum in tilt angles at \pm 25 - $30^{\circ}$ range of latitudes.

Table 3. Coefficients of latitudinal fit of Equation 1 (within the latitude range of \pm $10-25^{\circ}$ ) by solar cycle and maximum annual sunspot number

\begin{tabular}{r|rrr}
\hline Cycle & $\mathrm{A} \pm \sigma$ & $\mathrm{B} \pm \sigma$ & $\mathrm{SSN}$ \\
\hline 15 & $0.50 \pm 0.04$ & $-0.02 \pm 0.01$ & 175.7 \\
16 & $0.53 \pm 0.02$ & $-0.02 \pm 0.01$ & 130.2 \\
17 & $0.44 \pm 0.02$ & $0.03 \pm 0.01$ & 198.6 \\
18 & $0.49 \pm 0.03$ & $0.01 \pm 0.01$ & 218.7 \\
19 & $0.36 \pm 0.03$ & $0.02 \pm 0.01$ & 285.0 \\
20 & $0.47 \pm 0.01$ & $-0.00 \pm 0.00$ & 156.6 \\
21 & $0.50 \pm 0.03$ & $0.00 \pm 0.01$ & 232.9 \\
22 & $0.53 \pm 0.01$ & $0.02 \pm 0.00$ & 212.5 \\
23 & $0.53 \pm 0.01$ & $0.01 \pm 0.00$ & 180.3 \\
24 & $0.41 \pm 0.04$ & $0.00 \pm 0.01$ & 116.4 \\
\hline
\end{tabular}


The reader should note, however, that while we invoke the action of the Coriolis force to explain a non-monotonic behavior of active region tilts with latitude, we do not argue that this effect is the only explanation for the observed orientation of active regions. For example, Kosovichev and Stenflo (2008) see the relaxation of tilt after the active region emergence to some mean value for a given latitude (and not strictly to East-West direction) as an indication that tilt must represent the orientation of toroidal field in the convection zone. The functional dependence of tilt on sine of latitude fitted by Equation 2 should only be used for the range of latitudes hosting active regions $\left(\approx \pm 45^{\circ}\right)$. The reader could notice that at the latitude of about $64-65^{\circ}$ the fitted curve with cross zero and reverse its sign.

The presence of an offset in the non-zero tilt at solar equator is a clear indication that the Coriolis force alone cannot explain the active region tilt. The most plausible explanation is the kink instability inside the flux tubes that define the active regions. The direction of the kink would be determined by the sense of the internal twist (helicity) inside the magnetic flux tube. In fact, earlier investigations do find that for some active regions, the sign-relation between active region tilt and internal twist of their magnetic fields supports the interpretation of tilt as the result of kink-instability (Canfield and Pevtsov, 1998; Holder et al., 2004; Tian et al., 2005). There is also a possibility that the non-zero tilt at solar equator might be a consequence of the (sunspot) magnetic equator not being aligned with the solar rotation equator. The idea of sunspot-based magnetic equator was introduced by Pulkkinen et al. (1999), who defined it as an average latitude of sunspots of the northern and southern hemispheres. They also found the location of this sunspot magnetic equator to switch between the northern and southern hemisphere with a period of about 90 years. Zolotova et al. (2009) verified this hemispheric asymmetry in sunspot activity, with magnetic equator being offset to the south during Cycles 12-15, to the north during Cycles 1719 and back to the south during Cycles 20-23. However, since these results find that the sunspot equator oscillates at a period of several solar cycles, they cannot explain the present observations. Instead, we note of the hemispheric asymmetry of the streamer belt, which was found to oscillate in a 22-year cycle (Mursula and Zieger, 2001; Mursula, Hiltula, and Zieger, 2002). Accordingly, the streamer belt is displaced northward or southward in alternating cycles. The alternating occurrence of positive (negative) tilts at the equator in odd (resp. even) cycles may lead to a corresponding north-south displacement of the streamer belt later in the cycle. This possible connection will be elaborated later in more detail. Anyway, this is the only north-south asymmetric phenomenon close to the solar equator, which alternates systematically from cycle to cycle.

Finally, although our data do show some variations in the steepness of slope of the latitudinal variation of tilts between different cycles, we found no correlation between steepness of latitudinal dependence of tilt angles in Cycle $(n)$ and the strength of the following sunspot Cycle $(n+1)$. This lack of correlation is not surprising, as the strength of sunspot cycle is affected by several other factors, not only by the tilt of active regions. For example, prior conditions of polar field (its strength) determine how much magnetic flux is required for its reversal and rebuilding for the next cycle. In addition, the strength of polar field may be affected 
by the emergence of active regions with non-Hale polarity orientation (Yeates, Baker, and van Driel-Gesztelyi, 2015). Recent modeling indicates that emergence of a single "rogue" region in the right phase of solar cycle may significantly reduce the strength of polar field, and in the extreme case, it may even shutdown the solar cycle (Nagy et al.,2017). The effect of "rogue" active regions may weaken a more "deterministic" effect of tilt of active regions on strength of polar field.

Acknowledgments Work of individual co-authors was supported by their National grants and projects: the Academy of Finland to the ReSoLVE Centre of Excellence (project no. 272157), the Russian Foundation for Basic Research (RFBR, project 18-02-00098), the Russian Science Foundation (RSF, project 15-12-20001), and NASA's grant NNX15AE95G. The authors are members of international team on Reconstructing Solar and Heliospheric Magnetic Field Evolution Over the Past Century supported by the International Space Science Institute (ISSI), Bern, Switzerland. The National Solar Observatory (NSO) is operated by the Association of Universities for Research in Astronomy (AURA), Inc., under cooperative agreement with the National Science Foundation.

Disclosure of Potential Conflicts of Interest The authors declare that they have no conflicts of interest.

\section{References}

Babcock, H.W.: 1961, The Topology of the Sun's Magnetic Field and the 22-YEAR Cycle. Astrophys. J. 133, 572. DOI ADS [Babcock1961]

Baumann, I., Schmitt, D., Schüssler, M., Solanki, S.K.: 2004, Evolution of the large-scale magnetic field on the solar surface: A parameter study. Astron. Astrophys. 426, 1075. DOI ADS [Baumann.etal2004]

Cameron, R.H., Jiang, J., Schmitt, D., Schüssler, M.: 2010, Surface Flux Transport Modeling for Solar Cycles 15-21: Effects of Cycle-Dependent Tilt Angles of Sunspot Groups. Astrophys. J. 719, 264. DOI ADS [Cameron.etal2010]

Canfield, R.C., Pevtsov, A.A.: 1998, Helicity of Solar Active-Region Magnetic Fields. In: Balasubramaniam, K.S., Harvey, J., Rabin, D. (eds.) Synoptic Solar Physics, Astronomical Society of the Pacific Conference Series 140, 131. ADS [Canfield.Pevtsov1998]

Dasi-Espuig, M., Solanki, S.K., Krivova, N.A., Cameron, R., Peñuela, T.: 2010, Sunspot group tilt angles and the strength of the solar cycle. Astron. Astrophys. 518, A7. DOI ADS [Dasi-Espuig.etal2010]

Dasi-Espuig, M., Solanki, S.K., Krivova, N.A., Cameron, R., Peñuela, T.: 2013, Sunspot group tilt angles and the strength of the solar cycle (Corrigendum). Astron. Astrophys. 556, C3. DOI ADS [Dasi-Espuig.etal2013]

Fisher, G.H., Fan, Y., Howard, R.F.: 1995, Comparisons between theory and observation of active region tilts. Astrophys. J. 438, 463. DOI ADS [Fisher.etal1995]

Fisher, G.H., Fan, Y., Longcope, D.W., Linton, M.G., Pevtsov, A.A.: 2000, The Solar Dynamo and Emerging Flux - (Invited Review). Solar Phys. 192, 119. DOI, ADS [Fisher.etal2000]

Hale, G.E., Ellerman, F., Nicholson, S.B., Joy, A.H.: 1919, The Magnetic Polarity of Sun-Spots. Astrophys. J. 49, 153. DOI ADS [Hale.etal1919]

Holder, Z.A., Canfield, R.C., McMullen, R.A., Nandy, D., Howard, R.F., Pevtsov, A.A.: 2004, On the Tilt and Twist of Solar Active Regions. Astrophys. J. 611, 1149. DOI ADS [Holder.etal2004]

Howard, R.F.: 1991, Axial tilt angles of sunspot groups. Solar Phys. 136, 251. DOI ADS [Howard1991]

Ivanov, V.G.: 2012, Joy's law and its features according to the data of three sunspot catalogs. Geomagnetism and Aeronomy 52, 999. DOI ADS. [Ivanov2012] 
Kosovichev, A.G., Stenflo, J.O.: 2008, Tilt of Emerging Bipolar Magnetic Regions on the Sun. Astrophys. J. Lett. 688, L115. DOI ADS [Kosovichev.Stenflo2008]

Leighton, R.B.: 1969, A Magneto-Kinematic Model of the Solar Cycle. Astrophys. J. 156, 1. DOI ADS [Leighton1969]

Li, J., Ulrich, R.K.: 2012, Long-term Measurements of Sunspot Magnetic Tilt Angles. Astrophys. J. 758, 115. DOI ADS [Li.Ulrich2012]

Longcope, D., Linton, M., Pevtsov, A., Fisher, G., Klapper, I.: 1999, Twisted Flux Tubes and How They Get That Way. Washington DC American Geophysical Union Geophysical Monograph Series 111, 93. DOI. ADS. [Longcope.etal1999]

McClintock, B.H., Norton, A.A.: 2013, Recovering Joy's Law as a Function of Solar Cycle, Hemisphere, and Longitude. Solar Phys. 287, 215. DOI. ADS. [Mcclintock. Norton2013]

McClintock, B.H., Norton, A.A.: 2016, Tilt Angle and Footpoint Separation of Small and Large Bipolar Sunspot Regions Observed with HMI. Astrophys. J. 818, 7. DOI ADS [McClintock. Norton2016]

McClintock, B.H., Norton, A.A., Li, J.: 2014, Re-examining Sunspot Tilt Angle to Include Anti-Hale Statistics. Astrophys. J. 797, 130. DOI ADS [McClintock.etal2014]

Mursula, K., Zieger, B.: 2001, Long-term north-south asymmetry in solar wind speed inferred from geomagnetic activity: A new type of century-scale solar oscillation? Geophys. Res. Lett. 28, 95. DOI ADS [Mursula.Zieger2001]

Mursula, K., Hiltula, T., Zieger, B.: 2002, Latitudinal gradients of solar wind speed around the ecliptic: Systematic displacement of the streamer belt. Geophys. Res. Lett. 29, 1738. DOI ADS [Mursula.etal2002]

Nagy, M., Lemerle, A., Labonville, F., Petrovay, K., Charbonneau, P.: 2017, The Effect of "Rogue" Active Regions on the Solar Cycle. Solar Phys. 292, 167. DOI ADS [Nagy.etal2017]

Norton, A.A., Gilman, P.A.: 2005, Recovering Solar Toroidal Field Dynamics from Sunspot Location Patterns. Astrophys. J. 630, 1194. DOI ADS [Norton.Gilman2005]

Pevtsov, A.A., Clette, F.: 2014, To understand future solar activity, one has to know the past. EOS 98. DOI. [Pevtsov. Clette2017]

Pevtsov, A.A., Berger, M.A., Nindos, A., Norton, A.A., van Driel-Gesztelyi, L.: 2014, Magnetic Helicity, Tilt, and Twist. Space Sci. Rev. 186, 285. DOI ADS [Pevtsov.etal2014]

Press, W.H., Teukolsky, S.A., Vetterling, W.T., Flannery, B.P.: 1992, Numerical recipes in FORTRAN. The art of scientific computing. ADS [Press.etal1992]

Pulkkinen, P.J., Brooke, J., Pelt, J., Tuominen, I.: 1999, Long-term variation of sunspot latitudes. Astron. Astrophys. 341, L43. ADS [Pulkkinen.etal1999]

Sattarov, I., Pevtsov, A.A., Hojaev, A.S., Sherdonov, C.T.: 2002, X-Ray Bright Points and Photospheric Bipoles during Cycles 22 and 23. Astrophys. J. 564, 1042. DOI. ADS [Sattarov.etal2002]

Sivaraman, K.R., Gupta, S.S., Howard, R.F.: 1999, Measurement of Kodaikanal white-light images - IV. Axial Tilt Angles of Sunspot Groups. Solar Phys. 189, 69. DOI ADS [Sivaraman.etal1999]

Stenflo, J.O., Kosovichev, A.G.: 2012, Bipolar Magnetic Regions on the Sun: Global Analysis of the SOHO/MDI Data Set. Astrophys. J. 745, 129. DOI ADS. [Stenflo.Kosovichev2012]

Tian, L., Liu, Y., Yang, J., Alexander, D.: 2005, The Role of the Kink Instability of a LongLived Active Region AR 9604. Solar Phys. 229, 237. DOI ADS [Tian.etal2005]

Tlatov, A.G., Pevtsov, A.A.: 2010, The latitude of ephemeral regions as an indicator for solarcycle strength. Mem. S.A.It 81, 814. ADS [Tlatov.Pevtsov2010]

Tlatov, A.G., Vasil'eva, V.V., Pevtsov, A.A.: 2010, Distribution of Magnetic Bipoles on the Sun over Three Solar Cycles. Astrophys. J. 717, 357. DOI ADS [Tlatov.etal2010]

Tlatova, K.A., Vasil'eva, V.V., Pevtsov, A.A.: 2015, Long-term variations in the sunspot magnetic fields and bipole properties from 1918 to 2014. Geomagnetism and Aeronomy 55, 896. DOI ADS [Tlatova.etal2015]

Yeates, A.R., Baker, D., van Driel-Gesztelyi, L.: 2015, Source of a Prominent Poleward Surge During Solar Cycle 24. Solar Phys. 290, 3189. DOI ADS. [Yeates.etal2015]

Zirin, H.: 1988, Astrophysics of the sun. ADS [Zirin1988]

Zolotova, N.V., Ponyavin, D.I., Marwan, N., Kurths, J.: 2009, Long-term asymmetry in the wings of the butterfly diagram. Astron. Astrophys. 503, 197. DOI ADS [Zolotova.etal2009] 